\title{
On the collision of drops in turbulent clouds
}

\author{
By P. G. SAFFMAN and J. S. TURNER \\ Trinity College, Cambridge
}

(Received 16 November 1955)

\begin{abstract}
SUMMARY
This paper proposes a theory of collisions between small drops in a turbulent fluid which takes into account collisions between equal drops. The drops considered are much smaller than the small eddies of the turbulence and so the collision rates depend only on the dimensions of the drops, the rate of energy dissipation $\epsilon$ and the kinematic viscosity $\nu$. Reasons are given for believing that the collision efficiency for nearly equal drops is unity, and the collision rate due to the spatial variations of turbulent velocity is shown to be $N=1 \cdot 30\left(r_{1}+r_{2}\right)^{3} n_{1} n_{2}(\epsilon / \nu)^{1 / 2}$, valid for $r_{1} / r_{2}$ between one and two. A numerical integration has been performed using this expression to show how an initially uniform distribution will change because of collisions. An approximate calculation is then made to take account also of collisions which occur between drops of different inertia because of the action of gravity and the turbulent accelerations.

The results are applied to the case of small drops in atmospheric clouds to test the importance of turbulence in initiating rainfall. Estimates of $\epsilon$ are made for typical conditions and these are used to calculate the initial rates of collision, the change in mean properties and the rate of production of large drops. It is concluded that the effects of turbulence in clouds of the layer type should be small, but that moderate amounts of turbulence in cumulus clouds could be effective in broadening the drop size distribution in nearly uniform clouds where only the spatial variations of velocity are important. In heterogeneous clouds the collision rates are increased, and the effects due to the inertia of the drop soon become predominant. The effect of turbulence in causing collisions between unequal drops becomes comparable with that of gravity when $\epsilon$ is about $2000 \mathrm{~cm}^{2} \mathrm{sec}^{-3}$.
\end{abstract}

\section{INTRODUCTION}

It has been agreed for many years that while the initial process in the formation of clouds in the atmosphere must be one of condensation from the vapour phase, this process is not sufficiently rapid for the small water droplets to grow to raindrop sizes in the times usually available. Bergeron (1933) suggested that ice crystals might play a crucial part in the mechanism 
by which raindrops are formed, but observational evidence since that time has proved that heavy rain can fall from clouds whose temperatures are nowhere below the freezing point.

Various workers have therefore developed the idea of the growth of raindrops by the coalescence of liquid cloud drops. These theories suppose that drops larger than the mean drop size fall through the cloud under the action of gravity, and sweep up some of the smaller drops in their path. The rate of growth of the falling drops can be calculated and is found to be a rapidly increasing function of the size of the drop.

Squires (1952) has investigated theoretically the size distribution of cloud drops condensing on hygroscopic nuclei, and has concluded that only under rather special circumstances can a few droplets considerably larger than the mean be formed. If, for example, there are a few giant nuclei, these will quickly grow, by condensation, to such a size that the above coalescence mechanism will become important. Otherwise, the calculated drop size distribution is more uniform than that observed, as is also clear from the work of Howell (1949), and the mean size is smaller than that for which the above coalescence mechanism becomes more important than condensation.

A natural suggestion therefore is that turbulence in a cloud might lead to collisions among the drops, thus giving a greater spread of cloud drop sizes and providing the larger droplets on which raindrops could form. (Bowen (1950) has indeed considered the history of drops of double the mass of the rest of the drops, these drops being supposed formed by the random collision of two drops, but the mechanism of such collisions was not examined.) East \& Marshall (1954) have discussed the previous (mostly qualitative) suggestions which have been made about the role that turbulence could play in precipitation, and they propose a new theory in which the effect of random motions is regarded as being equivalent to the action of an increased gravitational field. It is concluded that turbulence could be important in a heterogeneous cloud if the random air acceleration is comparable with the acceleration due to gravity. It should be noted that the process pictured by these authors is still ineffective if the drops are small, and that they predict zero collision rates in homogeneous clouds.

It is the purpose of the present investigation to discuss a mechanism due to turbulence which gives collisions between equal drops and which employs a more realistic model of the nature of the turbulent motion than that used by East \& Marshall. The collision frequencies are found in terms of the rate of energy dissipation per unit mass in the cloud and this quantity is estimated from the large scale properties of the air motion inside the clouds.

\section{The NATURE OF THE TURBUlent MOtion}

East \& Marshall regarded the turbulence as equivalent in its effects to a random motion in time of the whole air parcel containing all the drops, and neglected the spatial variations which are surely an essential feature of

F.M. 
turbulent motion. All collisions on their model are therefore due to the different motion, relative to the air, of drops of different sizes; and hence they found that equal drops did not collide. The effect of the spatial variations, however, is to give neighbouring drops different velocities and thus cause collisions, whatever the ratio of the sizes of the drops.

The cloud droplets that we are considering are usually smaller by at least an order of magnitude than the length scale of the small eddies of the turbulence, and so the relative motion of two neighbouring drops will be governed by the small scale motion. It has been pointed out by Batchelor (1950) and others that because the Reynolds number for turbulent motion in the atmosphere is usually large, the similarity theory of turbulent motion will hold for the small scale motion. This theory implies that for scales of motion sufficiently small compared to the energy-containing eddies, the motion is isotropic and the mean values of quantities related to the turbulence will depend only on the kinematic viscosity $\nu$ and the rate of energy dissipation per unit mass $\epsilon$, provided that the quantities concerned depend strongly on the small scale properties of the turbulence. For example, the relative diffusion of a cloud of smoke can be treated in this way, whereas diffusion from a fixed source can not, since in the latter case the large eddies are also important. It follows that the effect of turbulence in causing collisions between neighbouring droplets will also depend on the rate of turbulent energy dissipation per unit mass $\epsilon$, and it will be necessary to make an estimate of this quantity in clouds under various conditions. We shall do this first before describing the mechanism of collisions.

Brunt (1939) has given as an average value in the lowest few kilometres of the atmosphere $\epsilon=5 \mathrm{~cm}^{2} \mathrm{sec}^{-3}$. Few direct measurements which allow $\epsilon$ to be estimated in clouds have been made, but R. J. Taylor (1952) has deduced values of $\epsilon$ of the order of $1000 \mathrm{~cm}^{2} \mathrm{sec}^{-3}$ close to the ground in moderate winds. Another approach to the problem is available however.

The results of laboratory experiments (e.g. Batchelor \& Townsend 1948) have indicated that the rate of turbulent energy dissipation is usually of order $u^{3} / l$, where $u$ is a root-mean-square turbulent velocity and $l$ is a length scale associated with the energy-containing eddies. Measurements of accelerations experienced by aircraft in bumpy conditions indicate that fluctuating velocities of a few metres per second are common, with corresponding eddy sizes of the order of tens or hundreds of metres. If we take as typical figures, $u=2$ metres $/ \mathrm{sec}, l=50$ metres, we obtain $\epsilon \doteqdot 1600 \mathrm{~cm}^{2} \mathrm{sec}^{-3}$. We might suppose that something less than the mean figure of $\epsilon=5 \mathrm{~cm}^{2} \mathrm{sec}^{-3}$ would be applicable to stratiform clouds where there is a small mean velocity, and take a larger value say $\epsilon=1000 \mathrm{~cm}^{2} \mathrm{sec}^{-3}$ as an estimate of the conditions in turbulent cumulus clouds. However, it may be possible to make better estimates than these for the different types of clouds.

\section{Discussion OF THE COLLISION PROCESS}

There are two ways in which turbulence causes collisions between neighbouring droplets. Firstly, there are the spatial variations of the 
turbulent motion referred to previously. Collisions due to this process can conveniently be called 'collisions due to the motion of the droplets with the air'. Secondly, each droplet is moving relative to the air surrounding it, owing to the fact that the inertia of a droplet is different from that of an equal volume of air. It follows that neighbouring droplets of unequal size will have different velocities (since the inertia of a droplet depends on its size), and this also will lead to collisions. This process is called 'collisions due to the motion relative to the air'. The latter process will not give collisions between droplets of equal sizes, such collisions being due to the first process.

Before we can calculate the rate at which collisions between droplets occur, it is necessary to consider the effect that the presence of a droplet has on the motion of neighbouring droplets, that is, we must consider the distortion of the flow due to the presence of a drop. A measure of this distortion is the collision efficiency, which can be defined as that proportion of drops which would have collided in the absence of distortion, actually to do so. It is clear that the collision efficiency must be dependent on the nature of the flow. Collision efficiencies were calculated by Langmuir (1948) for the case of small drops suspended in the steady laminar flow around a fixed large sphere. His numerical results are only likely to be accurate in cases where the basic assumptions are satisfied, although they have been extensively applied beyond their range of validity by others, and by East \& Marshall in particular. It is difficult to justify the application of Langmuir's results to the present problem, since we are concerned with a case in which the colliding drops are of nearly equal sizes. Recent measurements, by Telford, Thorndike \& Bowen (1955), of collection efficiencies for nearly equal drops somewhat larger than cloud drop sizes, have indeed given values considerably higher than would be predicted by an application of Langmuir's theory.

Now the Reynolds number of the relative motion of two nearly equal approaching cloud droplets will usually be much less than one, and some relevant experimental evidence for this range of Reynolds numbers is provided by the experiments of Manley \& Mason (1952, 1955). They observed the collisions between glass spheres and between air bubbles suspended in a uniformly sheared viscous liquid, and showed that in these circumstances, the collision efficiency is indeed unity, that is, the distortion of the flow does not influence the collision rate.

It thus seems that, in the absence of further evidence, it is not unreasonable to take the collision efficiency of nearly equal droplets as unity; this is equivalent to neglecting altogether the distortion of the flow by a drop.

We are interested primarily in collisions between nearly equal drops, and as a beginning we shall confine our attention to collisions due to motion with the air. Collisions due to motion relative to the air are not unimportant, but this process does not give collisions between equal drops. Later, we shall make an estimate of the relative importance of the two processes.

It is useful to keep in mind the qualitative picture of the local shearing motions which lead to collisions between drops carried along with the fluid. 
For two close points in a turbulent fluid, the relative motion is that of uniform strain. Taking an origin at the centre of one drop and the coordinate axes in the directions of the principal rates of strain, the streamlines of the relative motion in one of the coordinate planes are as shown in figure 1. The other drops are moving with the air along these streamlines and since we neglect the distortion of the flow field due to the presence of the drops, for the reasons mentioned above, the collision rate of the 'fixed drop' with other drops is just the flux of fluid inwards across the surface of a sphere, concentric with the fixed drop and of radius equal to the sum of the radii of the two approaching drops, multiplied by the number density of the other drops. The equivalent calculation for a uniform laminar flow assumes, of course, that all drops in a cylinder parallel to the flow and containing the effective cross-section of the fixed drop should meet that drop.

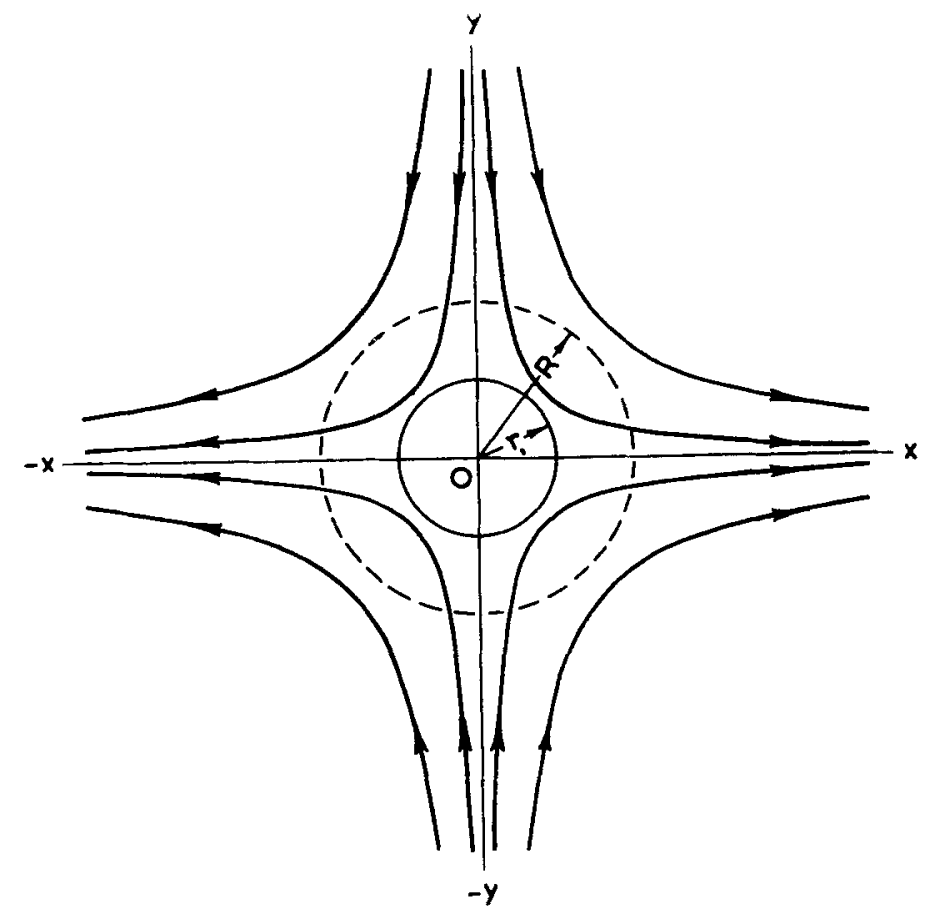

Figure 1. Streamlines of the relative motion in one of the principal planes.

\section{COLLISIONS BETWEEN DROPS MOVING WITH THE AIR}

We now proceed to the calculation of this collision rate. Let the mean concentrations of two sizes of drop in a given population be $n_{1}$ and $n_{2}$ per unit volume, and their radii $r_{1}$ and $r_{2}$ respectively. 'The collision radius for a pair of drops, one of each type, will be just the sum of the two radii, 
$R=r_{1}+r_{2}$ say. Now the mean flux of fluid into a sphere of radius $R$ surrounding one drop is

$$
-\overline{\int_{w_{r}<0} w_{r} d S}
$$

where $w_{r}$ is the radial component of the relative velocity, and the bar denotes a mean over many realizations of the motion. If we suppose the turbulence to be isotropic, this mean will be equal to the mean at a fixed point in space, since there is no correlation between the position of a drop and the properties of the turbulence. If the drops are randomly distributed and moving with the air, the collision rate is thus

$$
-n_{1} n_{2} \overline{\varlimsup_{w_{r}<0} w_{r} d S}
$$

and, by the similarity hypothesis, the integral will be a function of $\epsilon$ and $\nu$, and of the length $R$.

To evaluate the integral, we note that the equation of continuity of the fluid gives

$$
\int_{w_{r}<0} w_{r} d S+\int_{w_{r}>0} w_{r} d S=0
$$

Hence,

$$
-\overline{\int_{w_{r}<0} w_{r} d S}=\overline{\frac{1}{2} \int_{\text {entire sphere }}\left|w_{r}\right| d S}=\frac{1}{2} \int_{\text {entire sphere }} \overline{\left|w_{r}\right|} d S
$$

and since the small eddies are isotropic, the last expression becomes $2 \pi R^{2} \mid \overline{w_{x} \mid}$, where $w_{x}$ is the radial relative velocity along the radius parallel to the $x$-axis. Since $R$ is usually small compared with the length scale of the small eddies (as will be shown in $\S 6$ ), $\overline{\left|w_{x}\right|}=R \overline{|\partial u / \partial x|}$, where $u$ is the $x$-component of the velocity.

Further, the mean square of the velocity gradient is related to $\epsilon$ and $v$ through the expression $\left(\overline{\partial u / \partial x)^{2}}=\epsilon / 15 v\right.$ ('Taylor 1935). We now assume that $\partial u / \partial x$ is normally distributed and obtain $\overline{|\partial u / \partial x|}=(2 \epsilon / 15 \nu)^{1 / 2} . \quad(\partial u / \partial x$ is only approximately normally distributed but the error so introduced in the present context is not large; see Townsend (1947).) The collision rate is thus

$$
\begin{aligned}
N & =n_{1} n_{2}\left(r_{1}+r_{2}\right)^{3}\left(\frac{8 \pi \epsilon}{15 \nu}\right)^{1 / 2}=1 \cdot 30\left(r_{1}+r_{2}\right)^{3} n_{1} n_{2}\left(\frac{\epsilon}{v}\right)^{1 / 2} \\
& =\alpha_{12} n_{1} n_{2}, \text { say. }
\end{aligned}
$$

We can now use (1) to find the rate of growth of a population of equal drops. Let us suppose that initially we have a uniform size distribution of drops of type 1. Larger drops will form by multiple collisions, and we denote by $n_{s}$ the number of drops with $s$ times the mass of a type $1 \mathrm{drop}$. 
We also suppose that when two drops collide, they coalesce. Then the following equations hold (Smoluchowski 1917),

$$
\begin{aligned}
& \frac{d n_{1}}{d t}=-\alpha_{11} n_{1}^{2}-\alpha_{12} n_{1} n_{2}-\alpha_{13} n_{1} n_{3}-\ldots \\
& \frac{d n_{2}}{d t}=\frac{1}{2} \alpha_{11} n_{1}^{2}-\alpha_{12} n_{1} n_{2}-\alpha_{22} n_{2}^{2}-\alpha_{23} n_{2} n_{3}-\ldots \\
& \frac{d n_{3}}{d t}=\alpha_{12} n_{1} n_{2}-\alpha_{13} n_{1} n_{3}-\alpha_{23} n_{2} n_{3}-\alpha_{33} n_{3}^{2}-\ldots \\
& \frac{d n_{4}}{d t}=\alpha_{13} n_{13}+\frac{1}{2} \alpha_{22} n_{2}^{2}-\alpha_{14} n_{1} n_{4}-\alpha_{24} n_{2} n_{4}-\ldots
\end{aligned}
$$

Equations (2) can be solved in closed form if $\alpha_{i j}=2 \alpha$, that is, if it is assumed that the collision rate is independent of the particle size, and the solution is

$$
n_{s}=\frac{n_{0}\left(\alpha n_{0} t\right)^{s-1}}{\left(1+\alpha n_{0} t\right)^{s+1}}, \quad n=\frac{n_{0}}{1+\alpha n_{0} t},
$$

where $n$ is the concentration (total number of drops per unit volume) after time $t, n_{0}$ being the initial value. This solution was employed by Schumann (1940) in his study of the size distribution of fog particles, although he did not there discuss the effects of turbulence quantitatively. However, it is not satisfactory to neglect the dependence of collision rate upon drop size without further investigation and we therefore integrated equations (2) numerically. It was found that (3) can indeed lead to a serious underestimate of the number of large drops formed in a given time.

The equations (2) may be rewritten in a convenient dimensionless form if $n_{3} / n_{0}$ and $K=1 \cdot 30 t r_{1}^{3}(\epsilon / \nu)^{1 / 2} n_{0}$ are used as variables. Thus all the quantities which are given in a particular application are absorbed into the quantity $K$. The numerical integration was carried out over short intervals of $K=6 \times 10^{-3}$ by an iterative process, assuming a value for mean concentrations during the interval, working out the number of drops of each size produced and removed, and checking the assumed values.

The integration was continued until the total number of drops was reduced to two-thirds of the initial value, that is, until the mean drop mass increased by $50 \%$. At this stage, $K=1 \cdot 044 \times 10^{-1}$. The effect of multiple collisions in broadening the drop size distribution is shown in figure 2, where the results of our numerical integration are plotted. The numbers of drops of each size up to eight times the original mass are plotted on a logarithmic scale for three values of $K$. For comparison, the numbers calculated assuming a collision rate independent of size (i.e. using (3)), are also shown. (The lines in the figure are intended for guidance, and have no meaning in themselves.) It is clear that this approximation underestimates the number of eightfold drops present at a given time by a factor of twenty or more, and that the discrepancy increases with size. As a method for estimating the rate of change of the mean properties, the approximation is reasonable provided $K$ is not too large. 
Let us now use the result of the numerical integration to estimate the time necessary for the mean mass of cloud drops to increase by $50 \%$. We have $1.30 t r_{1}^{3}(\epsilon / \nu)^{1 / 2} n_{0}=1.044 \times 10^{-1}$ in c.g.s. units, or $w t(\epsilon / \nu)^{1 / 2}=3.36 \times 10^{5}$, where $w$ is the liquid water content in $\mathrm{gm} /$ cubic metre and $t$ is the time in

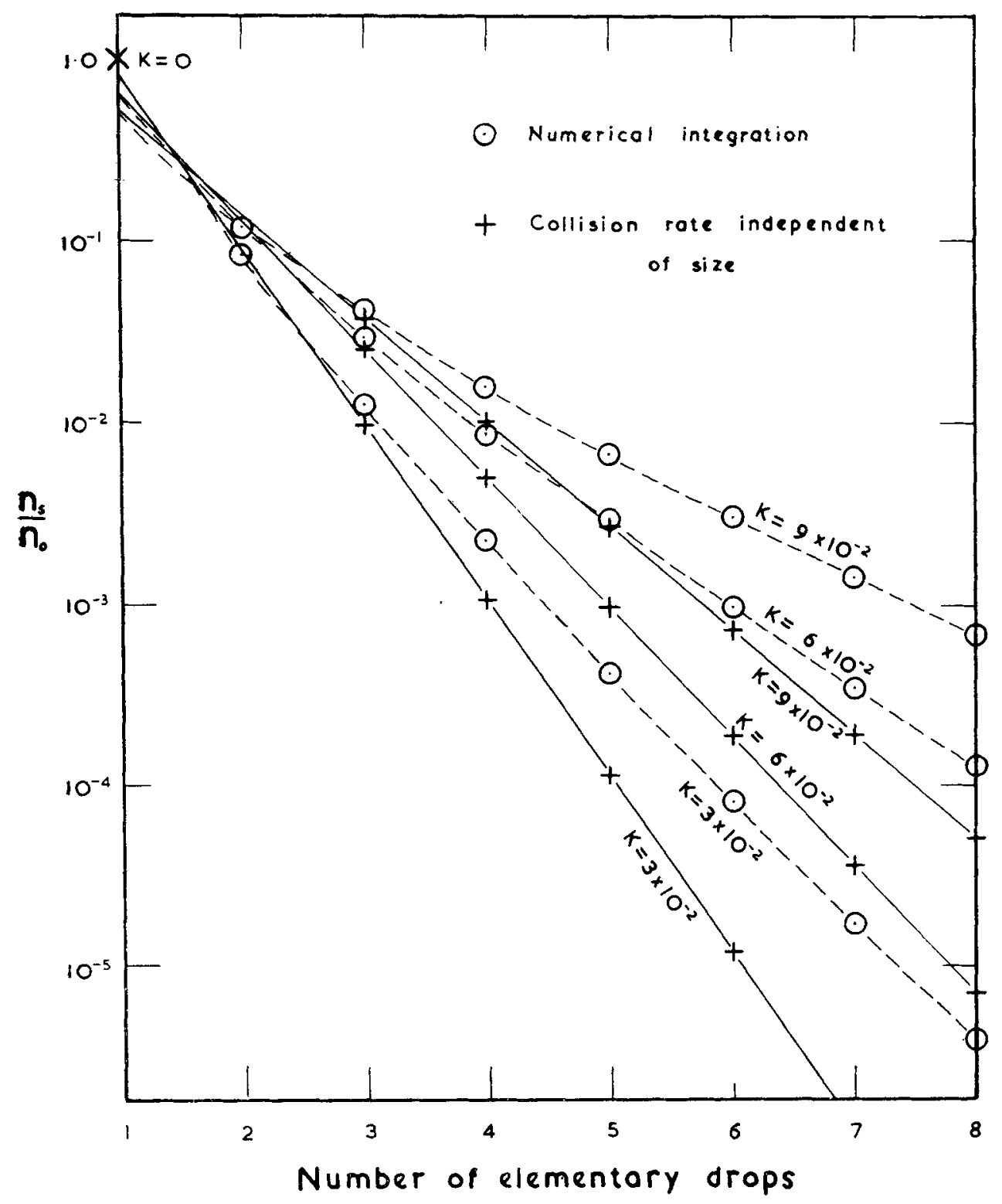

Figure 2. Distribution of drop sizes produced from an initially uniform population. Circles show the result of the numerical integration, and crosses show the result of a calculation assuming the collision rate to be independent of size. 
seconds. So with typical figures of $w=1.5 \mathrm{gm} /$ cubic metre and $\nu=0.17$ $\mathrm{cm}^{2} \mathrm{sec}^{-1}$, we obtain as the time for the mean mass to increase by $50 \%$, owing to the assumed motion with the air,

$$
\begin{aligned}
& t=4 \cdot 0 \times 10^{4} \text { seconds }=11 \text { hours, if } \epsilon=5 \mathrm{~cm}^{2} \mathrm{sec}^{-3}, \\
& t=2 \cdot 8 \times 10^{3} \text { seconds }=\frac{3}{4} \text { hour, if } \epsilon=1000 \mathrm{~cm}^{2} \mathrm{sec}^{-3} .
\end{aligned}
$$

Thus, the motion of the drops with the air will affect the mean properties of a cloud rather slowly, except under conditions of vigorous turbulence.

In applying these ideas to the initiation of rainfall, however, we are interested primarily not in how the average drop size changes, but in the rate at which a few large drops form. Let us, for example, see how frequently the double drops required by the Bowen theory will form. He assumed uniform drops of $10 \mu$ radius, and a liquid water content of $1 \mathrm{gm} / \mathrm{cubic}$ metre, corresponding to a concentration of $240 \mathrm{drops} / \mathrm{cm}^{3}$. The rate of production of double drops initially is then $3.0 \times 10^{-4}(\epsilon / \nu)^{1 / 2} \mathrm{~cm}^{-3} \mathrm{sec}^{-1}$. This shows that with $\epsilon=5 \mathrm{~cm}^{2} \sec ^{-3}, 0.04 \%$ of the total number are double drops after one minute, and with $\epsilon=1000 \mathrm{~cm}^{2} \mathrm{sec}^{-3}, 0.6 \%$ are double drops after one minute. Thus moderate amounts of turbulence should produce a significant concentration of double drops.

Again, if we apply the numerical results for multiple collisions to a case in which $n=600 \mathrm{drops} / \mathrm{cm}^{3}$ initially, $w=1.5 \mathrm{gm} / \mathrm{cubic}$ metre and $\epsilon=100$ $\mathrm{cm}^{2} \mathrm{sec}^{-3}$, we find that in 16 minutes there will be 100 drops per litre with mass equal to or greater than four times the original mass, and after 90 minutes, 100 drops per litre with mass equal to or greater than eight times the original. For sizes greater than this, the rates of growth increase rapidly, but so too does the relative importance of other processes, as will be seen in the following section.

\section{Motion Relative to the air}

In this section we shall consider approximately the motion of the drops both with the air and relative to the air, and also the relative motion of the drops due to gravity. In order to do this, we shall use a model of the collision process which will enable us to take all three processes into account at the same time. This will provide us with an indication of how the effects of the three processes should be added in a case in which they are calculated separately in some other way. It is to be noted, however, that the assumption that the collection efficiency is unity (see $\S 3$ ) is only likely to be valid for nearly equal drops, and so the following calculation will not apply to drops of very different sizes*.

The argument will proceed as follows. The probability distribution of the relative velocity of two drops is in general a function of their separation. When the separation of the centres of the drops is small compared with the

\footnotetext{
* The experiments of Manley \& Mason indicated a change in the relative motion of two colliding drops when the ratio of the larger radius to the smaller was greater than two. When the ratio was less than two, they observed the collision efficiency to be unity.
} 
length scale of the small eddies (it is shown in $\$ 6$ that in clouds the drops are usually small enough for this to be possible), this probability distribution can be deduced from the equation of motion of a drop and a knowledge of the statistical properties of the small eddies of the turbulence, provided we neglect the distortion of the flow by a drop for the reasons given in $\S 3$. We shall then have, in particular, the probability distribution of the relative velocity of two drops just before they collide. We denote by $P(\mathbf{w}) d \mathbf{w}$ the probability that the relative velocity $w$ of two drops just before they collide lies in the range $\mathbf{w}, \mathbf{w}+d \mathbf{w}$.

Let us calculate the rate of collision between drops of radius $r_{1}$ and $r_{2}$, whose number densities are $n_{1}$ and $n_{2}$ respectively, in terms of $P(\mathbf{w})$. A collision will take place when the separation of the centres of the drops is $R=r_{1}+r_{2}$. We shall consider the collisions that occur in a small interval of time $\delta t$, where $\delta t$ is small compared to the time scale of the small eddies, and we may suppose that the drops move in straight lines with constant velocity during the interval $\delta t$. For the purpose of the calculation, we may suppose one of the colliding drops, of type 1 say, to be at rest, the velocity of the other drop just before the collision being $w$. Then a collision will take place in the interval $\delta t$ if, at the beginning of the interval, the centre of the second drop was somewhere in a volume $\pi R^{2} w \delta t$, where $w=|\mathbf{w}|$. We now integrate over all values of $\mathbf{w}$ and multiply by the number density of drops of type 2 and thus obtain the number of collisions between the 'stationary' drop and the other drops in the time $\delta t$. On multiplying by the number density of drops of type 1 and dividing by $\delta t$, we obtain the collision rate as

$$
N=\pi R^{2} n_{1} n_{2} \iiint w P(\mathbf{w}) d \mathbf{w}
$$

It is permissible to multiply by the number density in the above manner only when the mean velocity of two colliding droplets is statistically independent of their relative velocity; for if this were not so, the local concentration of droplets would be related to the relative velocity. However, the mean velocity is controlled by the large energy-containing eddies and the relative velocity by the small eddies, and it is known that the large and small eddies are statistically independent when the Reynolds number of the turbulence is large (see Taylor 1938).

We see from equation (4) that the collision rate can be found as soon as $P(\mathbf{w})$ is known. $P(\mathbf{w})$ contains the effects of the spatial variations of velocity in the fluid (giving collisions due to the motion with the air), the turbulent accelerations (giving collisions due to the motion relative to the air), and gravity. It could be expressed formally as a complicated function of these three effects, but in that form it would not be possible to evaluate the integral in (4) in finite or simple terms. Now the variance of $w$ can be evaluated without difficulty from the equations of motion of the drop and

* Further details of the argument leading to (4) may be found in Chapman \& Cowling, Mathematical Theory of Non-Uniform Gases, Oxford University Press, 1952, pp. $60,89,90$. 
the statistical properties of the turbulence; and the method we shall use in order to avoid the above difficulty and to give integrals which we can evaluate, is to replace $P(\mathbf{w})$ by a simpler function which has the same variance. We shall justify this method by showing that, in the special cases when either motion with the air or gravity alone is effective, it does not lead to significant error.

Before discussing the explicit form that we shall take for $P(\mathbf{w})$, it is convenient to calculate the variance of $w$. To do this, we consider two droplets which have velocities $c_{1}$ and $c_{2}$ just before they collide. We denote by $u_{1}$ and $u_{2}$ respectively the 'undisturbed' velocities of the air surrounding these drops, so that the relative velocities between the drops and the air surrounding them are $q_{1}=c_{1}-u_{1}, q_{2}=c_{2}-u_{2}$. Further, $w=c_{2}-c_{1}$. It follows that the variance of $\mathbf{w}$ is given by

$$
\operatorname{var}(\mathbf{w})=\operatorname{var}\left(\mathbf{c}_{2}-\mathbf{c}_{1}\right)=\operatorname{var}\left(\mathbf{q}_{2}-\mathbf{q}_{1}\right)+\operatorname{var}\left(\mathbf{u}_{2}-\mathbf{u}_{1}\right),
$$

provided we suppose $u_{2}-u_{1}$ to be statistically independent of $\mathbf{q}_{1}$ and $\mathbf{q}_{2}$. The second term is easily calculated since

$$
\operatorname{var}\left(\mathbf{u}_{2}-\mathbf{u}_{1}\right)=R^{2}\left(\overline{\left.\frac{\partial \mathrm{u}}{\partial x}\right)^{2}}=5 R^{2}\left(\overline{\left.\frac{\partial u}{\partial x}\right)^{2}}=\frac{1}{3} R^{2} \frac{\epsilon}{v}\right.\right.
$$

in view of the isotropy of the small eddies.

To calculate the first term, we.use the equation of motion of a drop, which is

$$
\frac{d \mathbf{c}}{d t}=-\frac{1}{\tau}(\mathbf{c}-\mathbf{u})+\frac{\rho}{\rho_{0}} \frac{d \mathbf{u}}{d t}+\left(\frac{\rho}{\rho_{0}}-1\right) \mathbf{g}
$$

where $d / d t$ denotes differentiation following a drop, $\mathbf{g}$ is the acceleration due to gravity, $\tau$ is the relaxation time of the droplet $\left(\tau=2 r^{2} \rho_{0} / 9 \mu\right.$ for small spherical drops obeying Stokes' law), $r$ is the radius of a drop, $\rho_{0}$ is the density of the drops, $\rho$ that of the air, and $\mu$ the viscosity of the air. Hence,

$$
\frac{d \mathbf{q}_{1}}{d t}+\frac{1}{\tau_{1}} \mathbf{q}_{1}=\left(\frac{\rho}{\rho_{0}}-1\right)\left(\frac{d \mathbf{u}}{d t}+\mathbf{g}\right) .
$$

Now if $\tau$ is small compared with the time scale of the smallest eddies (and this will be shown in $\$ 6$ to be usually the case), we may neglect the first term on the left-hand side of $(7)$ and replace $\overline{(d \mathbf{u} / d t)^{2}}$ by $\overline{(D \mathbf{u} / D t)^{2}}$, and obtain

$$
\overline{\mathbf{q}_{1}^{2}}=\tau_{1}^{2}\left(1-\frac{\rho}{\rho_{0}}\right)^{2}\left[\overline{\left(\frac{d \mathbf{u}}{d t}\right)^{2}}+\mathbf{g}^{2}\right]=\tau_{1}^{2}\left(1-\frac{\rho}{\rho_{0}}\right)^{2}\left[3 \overline{\left(\frac{D u}{D t}\right)^{2}}+\mathbf{g}^{2}\right]
$$

We have already taken account of the relative motion of drops due to the difference between $u_{1}$ and $u_{2}$, so that in considering $q_{1}$ and $q_{2}$ we can suppose that the air velocities near two close particles are the same. (This is equivalent in effect to the assumption inherent in the work of East \& Marshall 
when they considered a random motion of the air parcel as a whole.) Thus, the correlation coefficient between $\mathbf{q}_{1}$ and $\mathbf{q}_{2}$ is unity and

Equation (5) then gives

$$
\operatorname{var}\left(\mathbf{q}_{2}-\mathbf{q}_{1}\right)=\left(\tau_{1}-\tau_{2}\right)^{2}\left(1-\frac{\rho}{\rho_{0}}\right)^{2}\left[3 \overline{\left(\frac{D u}{D t}\right)^{2}}+\mathbf{g}^{2}\right] .
$$

$$
\operatorname{var}(\mathbf{w})=3\left(1-\frac{\rho}{\rho_{0}}\right)^{2}\left(\tau_{1}-\tau_{2}\right)^{2} \overline{\left(\frac{D u}{D t}\right)^{2}}+\left(1-\frac{\rho}{\rho_{0}}\right)^{2}\left(\tau_{1}-\tau_{2}\right)^{2} g^{2}+\frac{1}{3} R^{2} \frac{\epsilon}{\nu} .
$$

We now return to the discussion of $P(\mathbf{w})$. We shall replace $P(\mathbf{w})$ by the function

$$
P *(\mathbf{w})=\left(\frac{\beta}{\pi}\right)^{3 / 2} \exp \left(-\beta \mathbf{w}^{2}\right)
$$

where $\beta$ is chosen so that the variance given by this distribution is the same as that for $P(\mathbf{w})$, that is, $\frac{3}{2} \beta^{-1}$ is equal to the expression (8). This seems to be the function that corresponds most closely to physical reality and yet renders the integration of (4) simple. If the relative velocity were due only to motion relative to the air, it seems likely that (9) would be accurate. Further, although the use of (9) replaces the constant relative velocity that would arise from the differential fall of droplets of different sizes under gravity alone by a random relative velocity with the same mean square, it will be seen below that the error so introduced is not large.

When we consider the relative velocity due to the spatial variations of velocity in the fluid, however, we find that the choice of the distribution (9) is not free from inconsistency. To see what is happening, let us suppose that the droplets are moving entirely with the air, that is, that the relative velocity due to gravity and motion relative to the air is zero. It seems that in this case (9) is inconsistent with the isotropy of the small eddies. For instance, if the separation of the two droplets is parallel to the $x$-axis, it follows from the isotropy that $\overline{\left(w_{x}\right)^{2}}=\frac{1}{2}\left(w_{y}\right)^{2}=\frac{1}{2}\left(\bar{w}_{z}\right)^{2}$; but according to equation (9), $\overline{\left(w_{x}\right)^{2}}=\overline{\left(w_{y}\right)^{2}}=\overline{\left(w_{z}\right)^{2}}$. However, despite this anomaly, the result obtained by the use of (9) will be shown to be not too different from that obtained in $\$ 4$, where the model used is consistent with the isotropy of the small eddies but still assumes the normal distribution of the separate components of the relative velocity.

We now use (9) to calculate the collision rate. On substituting in (5) and integrating, we obtain

$$
\begin{aligned}
N=\pi R^{2} n_{1} n_{2} \iiint w\left(\frac{\beta}{\pi}\right)^{3 / 2} \exp \left(-\beta \mathbf{w}^{2}\right) d \mathbf{w}=2 R^{2} n_{1} n_{2}\left(\frac{\pi}{\beta}\right)^{1 / 2} \\
=2(2 \pi)^{1 / 2} R^{2} n_{1} n_{2}\left[\left(1-\frac{\rho}{\rho_{0}}\right)^{2}\left(\tau_{1}-\tau_{2}\right)^{2} \overline{\left(\frac{D u}{D t}\right)^{2}}\right. \\
\left.\quad+\frac{1}{3}\left(1-\frac{\rho}{\rho_{0}}\right)^{2}\left(\tau_{1}-\tau_{2}\right)^{2} g^{2}+\frac{1}{9} R^{2} \frac{\epsilon}{\nu}\right]^{1 / 2} .
\end{aligned}
$$


The first two terms are zero when $\tau_{1}$ and $\tau_{2}$ are zero and the drops move with the air, when $\tau_{1}=\tau_{2}$ and the drops are identical, or when $\rho=\rho_{0}$ and the drops have the same density as the ambient fluid. When one of these is the case, (10) gives

$$
N=\frac{2}{3} R^{2} n_{1} n_{2}\left(\frac{2 \pi \epsilon}{\nu}\right)^{1 / 2}=1 \cdot 67 R^{3} n_{1} n_{2}\left(\frac{\epsilon}{v}\right)^{1 / 2} .
$$

It will be noticed that the constant here differs from that of equation (1), for the reasons mentioned above. Further, in the case when there is no turbulence, $(10)$ gives

$$
N=2\left(\frac{2 \pi}{3}\right)^{1 / 2} R^{2} n_{1} n_{2} g\left(1-\frac{\rho}{\rho_{0}}\right)\left|\tau_{1}-\tau_{2}\right|=2 \cdot 9 R^{2} n_{1} n_{2} g\left(1-\frac{\rho}{\rho_{0}}\right)\left|\tau_{1}-\tau_{2}\right|,
$$

whereas the exact calculation for this case, which is easily done, gives

$$
N=\pi R^{2} n_{1} n_{2} g\left(1-\frac{\rho}{\rho_{0}}\right)\left|\tau_{1}-\tau_{2}\right|
$$

Again we note that the difference in the constant is quite small.

\section{COMPaRison OF THE VARIOUS COLLISION MECHANISMS}

We now use (10) to compare the relative importance of the two turbulent collision mechanisms in clouds. It must first be shown that the radius $r$ and relaxation time $\tau=2 r^{2} \rho_{0} / 9 \mu$ of the droplets are usually small compared with the length scale $\left(\nu^{3} / \epsilon\right)^{1 / 4}$ and time scale $(\nu / \epsilon)^{1 / 2}$ of the small eddies, respectively, since the arguments leading up to (10) depend upon these conditions being satisfied. Now for drops of radius $7 \mu, \tau=6.3 \times 10^{-4}$ seconds, and for drops of radius $20 \mu, \tau=5.2 \times 10^{-3}$ seconds. The corresponding values of $\tau(\epsilon / \nu)^{1 / 2}$ and $r\left(\epsilon / \nu^{3}\right)^{1 / 4}$ are shown in the table. It can be seen that assumed conditions will be satisfied except perhaps for the largest drops in very strong turbulence.

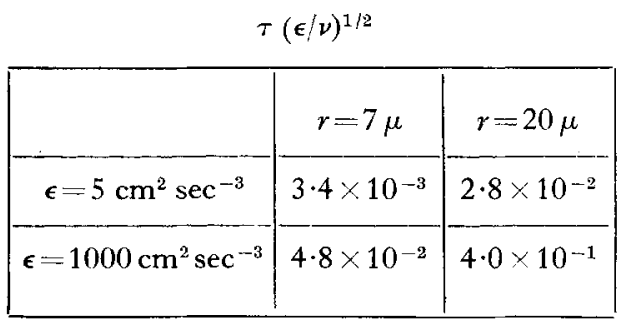

\begin{tabular}{|c|c|c|}
\hline & $r\left(\epsilon / \nu^{3}\right)^{1 / 4}$ \\
\hline & $r=7 \mu$ & $r=20 \mu$ \\
\hline$\epsilon=5 \mathrm{~cm}^{2} \mathrm{sec}^{-3}$ & $3.8 \times 10^{-3}$ & $1 \cdot 1 \times 10^{-2}$ \\
\hline$\epsilon=1000 \mathrm{~cm}^{2} \mathrm{sec}^{-3}$ & $1 \cdot 4 \times 10^{-2}$ & $4.0 \times 10^{-2}$ \\
\hline
\end{tabular}

The expression (10) may be evaluated in terms of known quantities by means of a result obtained by Batchelor (1951), who showed that

$$
\overline{\left(\frac{D u}{D t}\right)^{2}}=1 \cdot 3 v^{-1 / 2} \epsilon^{3 / 2}
$$

approximately when the Reynolds number of the turbulence is large. 
Hence, the ratio of the first term to the third in (10) is

$$
11 \cdot 7\left(1-\frac{\rho}{\rho_{0}}\right)^{2} \frac{(\nu \epsilon)^{1 / 2}}{R^{2}}\left(\tau_{1}-\tau_{2}\right)^{2}=8 \cdot 2 \times 10^{6} \epsilon^{1 / 2}\left(r_{1}-r_{2}\right)^{2},
$$

for water drops in air, where $r_{1}$ and $r_{2}$ are the drop radii in centimetres, the air viscosity has been taken as $1.72 \times 10^{-4}$ c.g.s. units, and $\rho$ as $1.00 \times 10^{-3}$ $\mathrm{gm} / \mathrm{cm}^{3}$ (values at $0^{\circ} \mathrm{C}$ and $800 \mathrm{mb}$ ). The ratio of the two terms is unity, that is, the effects are comparable when $r_{1}-r_{2}=2 \cdot 3 \mu$ for $\epsilon=5 \mathrm{~cm}^{2} \mathrm{sec}^{-3}$, and when $r_{1}-r_{2}=0.6 \mu$ for $\epsilon=1000 \mathrm{~cm}^{2} \mathrm{sec}^{-3}$.

Thus, except for the very small cloud droplets, the collision rates will increase to several times the values in $\$ 4$ when one drop is a few times the mass of the other; and eventually the collisions due to turbulence in heterogeneous clouds will be dominated by the motion relative to the air. (It should be emphasized again that the theory developed here is not likely to remain valid when the droplets become very different in size.)

It is also clear from (10) that the motion due to gravity and the motion of the drops relative to the air due to turbulence become comparable in their effects when the root-mean-square acceleration is of the same order as the acceleration due to gravity $g$; using (10) the condition for equality of the effects is $g \doteqdot 2 \nu^{-1 / 4} \epsilon^{3 / 4}$, which corresponds to $\epsilon \doteqdot 2100 \mathrm{~cm}^{2} \mathrm{sec}^{-3}$. Thus what we have called vigorous turbulence, with $\epsilon=1000 \mathrm{~cm}^{2} \mathrm{sec}^{-3}$, would be less effective than gravity in causing collisions.

Reviewing the results then, we have shown that if the low collection efficiencies calculated for uniform streaming and two drops of very different sizes are rejected as irrelevant for the case of nearly equal drops, considerable rates of coalescence are predicted in turbulent air, even in clouds with very uniform size distributions. In this latter case, the first collisions are due to the drops moving with the air, a process which does not seem to have been taken into account in previous theories. Particularly for the small drop sizes, where condensation is also important, the collisions of nearly equal drops concurrently with the condensation could lead to a broadening of the distribution of cloud drop sizes. As the size increases, and the range of sizes becomes larger, collisions due to the different relative motions between drops and the air (induced by the turbulence and a steady fall under gravity) will become predominant. Our theory does not allow us to follow the growth of a drop right up to raindrop sizes.

The magnitudes estimated for the initial collision rates are such that the properties of stratiform clouds would be affected rather slowly, whereas in a vigorous cumulus updraught the rate of production of larger drops might be sufficient to initiate the formation of rain. Thus we are not faced with the embarrassment of predicting that all clouds should fall out as rain.

The authors wish to express their thanks to Dr G. K. Batchelor for his suggestion of this problem, and for many helpful discussions. The work was made possible for one of us (P. G. S.) by the award of a Department of Scientific and Industrial Research maintenance grant, and for the other (J. S. T.) by the tenure of an Exhibition of 1851 Overseas Scholarship. 


\section{REFERENCES}

Batchelor, G. K. 1950 Quart. F. R. Met. Soc. 76, 133.

Batchelor, G. K. 1951 Proc. Camb. Phil. Soc. 47, 359.

Batchelor, G. K. \& Townsend, A. A. 1948 Proc. Roy. Soc. A, 193, 539.

Bergeron, T. 1933 Proc. Verb. de l'U.G.G.I. Lisbon, p. 156.

Bowen, E. G. 1950 Austr. F. Sci. Res. A, 3, 193.

East, T. W. R. \& Marshall, J. S. 1954 Quart. F. R. Met. Soc. 80, 26.

Howell, W. E. 1949 F. Met. 6, 134.

Langmuir, J. 1948 F. Met. 5, 175.

Manley, R. St. J. \& Mason, S. G. 1952 F. Coll. Sci. 7, 354.

Manley, R. ST. J. \& Mason, S. G. 1955 Can. F. Chem. 33, 763.

Schumann, T. E. W. 1940 Quart. F. R. Met. Soc. 66, 195.

Smoluchowski, M. 1917 Z. Phys. Chem. 92, 129.

Squires, P. 1952 Austr. F. Sci. Res. A, 5, 59, 473.

TaYlon, G. I. 1935 Proc. Roy. Soc. A, 151, 429.

TAYLOR, G. I. 1938 Proc. Roy. Soc. A, 164, 476.

TAYLOR, R. J. 1952 Quart. F. R. Met. Soc. 78, 179.

Telford, J. W., Thorndike, N. S. \& Bowen, E. G. 1955 Quart. Ұ. R. Met. Soc. 81, 241.

Townsend, A. A. 1947 Proc. Camb. Phil. Soc. 43, 560. 